$22 \mid 2016$

NOVECENTO... E DINTORNI

France et Italie (1955-1967) : politique, société et économie

\title{
Nicola Chiaromonte, «Tempo presente» e la 'crisi' della Francia
}

Nicola Chiaromonte, Tempo presente et la «crise " de la France

Nicola Chiaromonte, Tempo presente and the "crisis" of France

\section{Cesare Panizza}

\section{OpenEdition \\ Journals}

Edizione digitale

URL: http://journals.openedition.org/cei/3029

DOI: 10.4000/cei.3029

ISSN: 2260-779X

\section{Editore}

UGA Éditions/Université Grenoble Alpes

\section{Edizione cartacea}

Data di pubblicazione: 20 avril 2016

Paginazione: 203-217

ISBN: 978-2-84310-323-0

ISSN: $1770-9571$

Notizia bibliografica digitale

Cesare Panizza, «Nicola Chiaromonte, «Tempo presente» e la 'crisi' della Francia», Cahiers d'études italiennes [Online], 22 | 2016, online dal 01 janvier 2017, consultato il 26 mars 2021. URL: http:// journals.openedition.org/cei/3029; DOI: https://doi.org/10.4000/cei.3029 


\title{
NICOLA CHIAROMONTE, «TEMPO PRESENTE» E LA ‘CRISI’ DELLA FRANCIA
}

\author{
Cesare Panizza \\ Università degli Studi di Torino
}

\section{La copertina del primo numero di "Tempo presente»" la rivista fondata da Nicola Chiaromonte ${ }^{2}$ assieme a Ignazio Silone ${ }^{3}$ nel 1956, annunciava}

I. Su «Tempo presente», G. Fofi, V. Giacopini e M. Nonno (a cura di), Nicola Chiaromonte, Ignazio Silone. L'eredità di "Tempo presente», Roma, Fahrenheit 451, 2000; A. Donno, La cultura americana nelle rivista italiane del dopoguerra: "Tempo presente» (1956-1968), Lecce, Milella, 1978; P. Carlucci, "Tempo presente» (19561968) e il Congress for Cultural Freedom: alcuni appunti per la storia di una rivista, in D. Menozzi, M. Moretti e R. Pertici (a cura di), Culture e libertà: studi di storia in onore di Roberto Vivarelli, Pisa, Edizioni della Normale, 2006, pp. 453-478.

2. Per la biografia di Nicola Chiaromonte (Rapolla, Potenza, I905; Roma, 1972), G. Bianco, Nicola Chiaromonte e il tempo della malafede, Manduria-Bari-Roma, Lacaita, 1999; P. Craveri, Nicola Chiaromonte, in Dizionario biografico degli italiani, vol. 24, Roma, Istituto dell'Enciclopedia italiana, 1980, p. 600. Cfr. anche G. Fof, V. Giacopini e M. Nonno (a cura di), Nicola Chiaromonte, Ignazio Silone, cit., in cui compaiono saggi su Chiaromonte di G. Fofi, V. Giacopini, G. Gaeta, F. Bellincanta, F. La Porta, M. Sinibaldi; la sezione dedicata a Chiaromonte nel numero di agosto-settembre $201 \mathrm{I}$ di «Lo Straniero», vol. XV, n ${ }^{\circ}$ 134-I35, Nicola Chiaromonte nel tempo della malafede, con saggi di F. Bellincanta, M. Bresciani, M. Cicala, F. De Core, S. Fedele, G. Fofi, G. Gaeta, V. Giacopini, N. Lagioia, C. Panizza, G. Sumner, A. Tricomi; P. Adamo, "La prima cosa è dire no!»: Nicola Chiaromonte tra ragione, storia e utopia, in Dedicato a Nicola Chiaromonte nel trentennale della morte, "Quaderni dell'Altra tradizione», Forlì, Una città, 2002; V. Giacopini, Nicola Chiaromonte: una solitudine senza isolamento, in Id., Scrittori contro la politica, Torino, Bollati Boringhieri, 1999; F. La Porta, Le evidenze del mondo. Nicola Chiaromonte (1905-1972), in Maestri irregolari, Torino, Bollati Boringhieri, 2007; le singole introduzioni ai volumi che raccolgono i suoi scritti: quella di M. McCarthy a Scritti sul teatro, a cura di M. Chiaromonte, Torino, Einaudi, I975; di L. Valiani a Scritti politici e civili, a cura di M. Chiaromonte, Milano, Bompiani, 1976; di G. Herling a Il tarlo della coscienza, a cura di M. Chiaromonte, Bologna, Il Mulino, I972; di G. Pampaloni a Credere e non credere, a cura di M. Chiaromonte, Bologna, Il Mulino, I993 (I97I); di W. Karpinski a Che cosa rimane. Taccuini I955-I97I, a cura di M. Chiaromonte, Bologna, Il Mulino, I995; di S. Fedele a Le verità inutili, a cura dello stesso Fedele, Napoli, L'Ancora, 200I.

3. Silone e Chiaromonte si erano conosciuti nella primavera del 1934 in Svizzera. Avvicinatosi temporaneamente a Tasca dopo la rottura con Rosselli, Chiaromonte aveva cercato vanamente di coinvolgere lo scrittore abruzzese in una iniziativa comune. Negli anni successivi erano rimasti in stretto contatto, partecipando ad iniziative comuni, fra cui le attività fondatrici del Congresso per la libertà della cultura. Cfr. S. Soave, Senza tradirsi, senza tradire. Silone e Tasca dal comunismo al socialismo cristiano (1900-1940), Torino, Aragno, 2005, p. 303 e sgg.; sull'amicizia fra Silone e Chiaromonte, S. Pugliese, Bitter Spring. A life of Ignazio Silone, New York, Farrar, Strauss and Giroux, 2009, pp. 239-50. Più in generale per la biografia di Silone si rimanda a O. Gurgo e F. De Core, Silone. L'avventura di un uomo libero, Venezia, Marsilio, 1998. 
ben due articoli di autori francesi: La donna adultera ${ }^{4}$, un racconto breve di Albert Camus, uno dei 'numi tutelari' della rivista, e Colonialismo e coscienza tranquilla. Il dramma algerino, di Jean Daniel. Ancor più significativamente, Chiaromonte apriva la serie dei suoi interventi in Gazzetta - una sezione fissa della rivista che avrebbe accolto nei dodici anni successivi, e cioè fino alla chiusura di «Tempo presente» nel 1968, le sue annotazioni sull'attualità politica e culturale - proprio con un commento sulla situazione politica francese. Su quella nota, un trafiletto privo di titolo, è bene soffermarsi perché in poche righe vi si ritrovano sintetizzate le forme nelle quali Chiaromonte era solito valutare quanto accadeva oltralpe. Riprendendo implicitamente la riflessione di Daniel sulla "crisi della Francia», egli osservava come in fondo il dramma algerino non fosse causa, ma effetto dell'immobilismo che aveva quasi cristallizzato la vita politica e sociale della Quarta Repubblica. Era un' «inerzia» dovuta alla divisione dell'Europa in blocchi ideologicamente contrapposti, una realtà di cui la Francia risentiva certamente più di altri paesi europei. $\mathrm{Ne}$ era derivato un impulso diffuso a sottrarsi a quella logica bipolare, coltivato - talvolta in forme «nobili» ma in fondo sempre velleitarie — dalla maggior parte degli intellettuali "progressisti» francesi. Se ne era avuta una dimostrazione con il dibattito sul neutralismo degli anni precedenti che - va ricordato - Chiaromonte aveva puntualmente seguito nelle corrispondenze inviate da Parigi al «Mondo» di Mario Pannunzios. Quel malessere si era risolto in una sorta di 'provincialismo', nella «tentazione di ridurre la Francia dentro i suoi confini», giustificata negli intellettuali progressisti da quella equazione - così diffusa nella sinistra francese che vorrebbe coincidenti il progresso della Francia con quello del genere umano tout court. La riluttanza a prendere atto dei cambiamenti avvenuti con due guerre mondiali — la fine della centralità dell'Europa e della possibilità di ricondurre nella dimensione dello stato nazionale i problemi del presente, compreso quello della natura e della qualità effettiva dei sistemi democratici - gli pareva il sintomo più evidente di quella crisi, morale e culturale prima che politica — della 'coscienza europea' che durava da più di un secolo. Era una costellazione problematica di trasformazioni,

4. È molto probabilmente la prima edizione italiana del racconto di Camus, La femme adultère, pubblicato in Francia nel 1954 e poi nel 1957 raccolto insieme ad altri cinque nell'antologia L'exil et le royaume. La scelta non era casuale, visto che il racconto, al di là dei temi che affronta, è ambientato in Algeria.

5. Chiaromonte collaborò al «Mondo» di Pannunzio — di cui era amico dai tempi della giovinezza romana dalla sua fondazione. Dal 1949 al 1952 con una serie di corrispondenze da Parigi e da New York, sull'attualità politica e culturale, e al suo rientro in Italia, dal 1953 alla chiusura del settimanale nel 1966, in qualità di critico teatrale. 
quella sottesa al tema della crisi di civiltà, che Chiaromonte — come altri della generazione formatasi negli anni Venti - aveva lucidamente analizzato, ravvisandovi la matrice dei totalitarismi novecenteschi ${ }^{6}$. Eppure, quel modo di pensare e di collocarsi nel mondo era comunque degno di considerazione, in quanto il fatto, "che una nazione si identifichi con un'idea» - quella per Chiaromonte tanto controversa di progresso — «è un fatto grande e raro. Finisce coll'essere un destino» ${ }^{7}$.

Questa apparente contraddizione nel suo ragionamento trovava in realtà una sua spiegazione su un altro piano, di natura, si potrebbe dire, emozionale. Nell' incipit dell'articolo, Chiaromonte aveva infatti sostenuto che «i mali della Francia ci toccano come non ci toccano, malgrado tutto, i guai dell'Inghilterra in Medio Oriente; e ci interessano mentre l'attuale prosperità dei tedeschi ci lascia freddi» ${ }^{8}$. Non era solo agli italiani in genere che riferiva quelle parole, attingendo a un topos consueto, quello del reciproco rispecchiarsi dei due paesi l'uno nelle vicende dell'altro. Vi si celava qualcosa di più personale, un dato della sua stessa biografia. Se ne trova una chiara spiegazione in una lettera inviata da New York nel giugno del I946 ad Andrea Caff, il suo «unico maestro»e il suo «più caro amico»?. Nell'istituire un paragone fra la sua condizione di esule negli Stati Uniti

6. Su questa analisi che costituì il contributo più rilevante offerto da Chiaromonte all'elaborazione ideologica portata avanti dal movimento di Giustizia e Libertà con la pubblicazione dei «Quaderni di Giustizia e Libertà» (1932-1935) e poi del settimanale "Giustizia e Libertà», si veda C. Panizza, La morte si chiama fascismo. L'analisi del fascismo di Nicola Chiaromonte, "Quaderno di storia contemporanea", $\mathrm{n}^{\circ} 36,2004$, pp. 62-83; M. Bresciani, Cassandra a Parigi, "Lo Straniero», vol. XV, nº I34-I35, agosto-settembre 20II, pp. 57-72. Vale la pena ricordare che alla 'cultura della crisi' Chiaromonte apparteneva appieno, essendosi formato giovanissimo nel cenacolo filosofico di Adriano Tilgher e avendovi cercato una risposta nella riflessione filosofica (in particolare in quegli anni nell'opera di Husserl).

7. N. Chiaromonte, Gazzetta, «Tempo presente», vol. I, n I, I956, p. 50.

8. Ibid.

9. Chiaromonte conobbe Andrea Caffi nel 1932, durante il suo primo soggiorno parigino. A presentarglielo fu il comune amico Alberto Moravia. Ne nacque un sodalizio intellettuale e umano che nonostante il progressivo divaricarsi delle loro posizioni politiche negli anni della Guerra fredda, si rivelò per entrambi preziosissimo. Insieme a Renzo Giua e a Mario Levi — quelli che Aldo Garosci nella sua vita di Carlo Rosselli avrebbe definito $\mathrm{i}$ «novatori» - Caffi e Chiaromonte costituirono un vero e proprio cenacolo intellettuale interno a "Giustizia e Libertà", il movimento a cui tutti, a titolo diverso, collaboravano, destinato a scontrarsi, come appunto avvenne nel 1936, con la personalità di Carlo Rosselli. Su Caffi, M. Bresciani, La rivoluzione perduta. Andrea Caffi nella storia del Novecento, Bologna, Il Mulino, 2009; G. Bianco, Un socialista irregolare: Andrea Caffi intellettuale e politico d'avanguardia, Cosenza, Lerici, 1977; G. Landi (a cura di), Andrea Caffi. Un socialista libertario, Atti del convegno di Bologna (7 novembre 1993), Pisa, Bibiblioteca Franco Serantini, 1993; e la voce Andrea Caffi di Carlo Vallauri nel XVI volume del Dizionario biografico degli italiani, pp. 264-268. Sui «novatori», A. Garosci, Vita di Carlo Rosselli, cit., pp. 97-IO2; P. Bagnoli, Rosselli, Gobetti e la rivoluzione democratica. Uomini e idee tra liberalismo e socialismo, Firenze, La Nuova Italia, 1996, pp. 6I-I09; N. Ginzburg, Lessico famigliare, Torino, Einaudi, 1963, passim. Su Mario Levi, anche P. Gabrielli, Col freddo nel cuore. Uomini e donne nell'emigrazione antifascista, Donzelli, Roma, 2004, p. 147 e sgg. Su Renzo Giua, Giua Renzo di G. Sircana, in Dizionario Biografico degli Italiani, Istituto della Enciclopedia Italiana, Catanzaro, Arti Grafiche Abramo, 200I, pp. 65I652, e nelle memorie della sorella, L. Foa, É andata cosi, Palermo, Sellerio, 2005, p. 22. 
— dove si era rifugiato nel I94I e dove avrebbe vissuto fino al I948 - e il suo precedente esilio in Francia (dove Caffi viveva), Chiaromonte affermava che la Francia "n'avait jamais été terre d'exil pour moi» ${ }^{10}$. Non poteva affermare lo stesso degli USA, nonostante vi avesse conosciuto - dopo le inevitabili difficoltà iniziali — una stagione felice dal punto di vista intellettuale e umano, fatta per la prima volta anche di una certa stabilità, grazie a un relativamente agevole inserimento negli ambienti culturali ed editoriali newyorchesi. Cosa che non gli era invece mai occorsa in Francia, dove aveva vissuto fra il 1934 e il I94I, nonostante già in quella fase giovanile potesse contare su relazioni anche influenti.

A Parigi — stando a quanto ne scrisse lo stesso Chiaromonte - non aveva infatti mai conosciuto quella sensazione di straniamento che così sovente connota l'esperienza dell'esilio e che aveva invece caratterizzato i suoi anni americani. E non solo per la presenza di 'compagni' e amici, e di una vasta comunità di 'senzapatria' di ogni provenienza, che d'altronde certo a New York non mancavano, ma soprattutto per la sensazione di appartenere a quei luoghi, o meglio a quella civiltà, che fra tutti i popoli europei gli sembrava testimoniasse nelle sue espressioni e tradizioni migliori la più genuina e spontanea adesione ai valori dell'umanesimo.

Non fu certo un caso che al suo ritorno definitivo in Europa Chiaromonte scegliesse nel 1948 di ristabilirsi non a Roma, ma proprio a Parigi - dove rimase fino al 1953 - accettando il lavoro offertogli presso la sede centrale dell'Unesco. A deciderlo per la Francia furono sì la delusione per la vita intellettuale italiana del primo dopoguerra ma anche la forza dei legami, intellettuali e umani, intrecciati nella capitale francese negli anni duri dell'esilio antifascista.

La mappatura dei rapporti di Nicola Chiaromonte con gli intellettuali francesi nel corso degli anni è un lavoro che esula da questo contributo. Accenneremo brevemente soltanto ad alcune di queste relazioni, quelle indubbiamente più rilevanti. All'epoca del suo primo soggiorno parigino - un soggiorno di pochi mesi, avvenuto fra la fine del I93I e la primavera del 1932, ma rivelatosi decisivo perché Chiaromonte grazie all'incontro con Andrea Caffi e con Carlo Rosselli vi maturò la decisione di un'opposizione attiva al fascismo nelle fila del movimento Giustizia e

Io. Lettera di Nicola Chiaromonte ad Andrea Caffi, Truro, Massachussetts, 26 agosto 1946, fondo Andrea Caff, biblioteca Gino Bianco, Fondazione Alfred Lewin, Forlì. Ora riprodotta in M. Bresciani (a cura di), "Cosa sperare?». Il carteggio tra Andrea Caffi e Nicola Chiaromonte: un dialogo sulla rivoluzione (I932-1955), Napoli, Edizioni Scientifiche Italiane, 20I2, pp. 317-318. 
Libertà - deve essere fatta risalire la sua amicizia con André Malraux ${ }^{\text {II }}$. Un legame nato dall'ammirazione del giovane intellettuale italiano per il Malraux scrittore dominato dal «demone dell'azione» e che lo avrebbe indotto, nell'agosto del 1936, a partecipare all'impresa spagnola della squadriglia aerea España ${ }^{\mathrm{I} 2}$. Fu però proprio la Spagna e la diversa valutazione del peso assunto nel campo repubblicano dai comunisti e dall'Unione Sovietica - con conseguenze per Chiaromonte esiziali per le sorti della Repubblica - a determinare una rottura fra i due che le successive evoluzioni politiche di Malraux non avrebbero certo permesso di ricomporre. Una testimonianza di quei contrasti, ma anche della rilevanza per Malraux di quel rapporto di amicizia, è contenuta nelle pagine dell'Espoir dove Chiaromonte ha inspirato il personaggio dell'italiano Scali.

Alla perigliosa fuga di Chiaromonte dalla Francia, in direzione degli USA, nell'estate del I94I si dovette invece l'amicizia fra questi e Albert Camus, conosciuto a Algeri nell'attesa di potersi imbarcare, via Casablanca, verso le coste americane ${ }^{13}$. È forse il caso di ricordare che Chiaromonte ebbe un ruolo chiave nel far conoscere il pensiero e l'opera di Camus negli ambienti intellettuali newyorchesi a cui si era legato durante la sua permanenza negli Stati Uniti, in particolare attraverso la rivista "politics», diretta da Dwight Macdonald, cui anche lo scrittore francese collaborò per suo tramite ${ }^{\mathrm{I}}$. E che ebbe un ruolo di primo piano nell'organizzare il famoso viaggio che il direttore di «Combat» fece negli Stati Uniti nel 1946 per pronunziarvi, alla Columbia University, la celebre conferenza «The

II. Di Malraux, con cui era entrato in contatto per via epistolare ancor prima del suo arrivo a Parigi, come testimonia la corrispondenza fra i due conservata presso la Beinecke Library dell'Università di Yale dove sono state versate le sue carte, Chiaromonte scrisse a lungo e a più riprese, a partire da due saggi dedicatigli sulle pagine di «Solaria», Idee e figure di André Malraux e André Malraux e "La condition humaine», apparsi rispet-

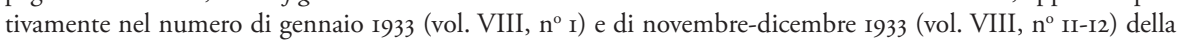
rivista fiorentina, fino al capitolo dedicatogli in Credere e non credere (apparso nel I97I, pochi mesi prima della morte, per Bompiani), André Malraux e il demone dell'azione, riformulazione di un saggio apparso nel numero di luglio del 1948 della newyorchese Partisan Review con il titolo André Malraux and the Demons of Actions e riproposto nel numero di agosto del 1960 di «Tempo presente». Si noti che oggi il lettore francese può usufruire della traduzione di Credere e non credere, recentemente pubblicata con un'introduzione di Marco Bresciani e una prefazione di Adam Michnik, nella serie dei Cahiers de l'Hôtel de Gallifet, Edizioni dell'Istituto italiano di Cultura, Parigi, 2013, che ha ripreso nel titolo - Le paradoxe de l'histoire - quello dell'edizione in lingua inglese del libro di Chiaromonte (The Paradox of History) apparsa un anno prima di quella italiana per Weidenfeld and Nicholson.

I2. Sulla partecipazione di Nicola Chiaromonte alla guerra civile spagnola, C. Panizza, Nicola Chiaromonte e la guerra civile spagnola, "Memoria e Ricerca», vol. XX, n 39, 20I2, pp. I57-I74.

13. Chiaromonte ha ricordato la nascita della loro amicizia in occasione della morte dello scrittore francese, Albert Camus, "Tempo presente», gennaio I960, ora in N. Chiaromonte, Il tarlo della coscienza, cit., pp. 2I7-222.

I4. Su "politics» e su Dwight Macdonald cfr. G. Sumner, Dwight Macdonald and the "politics» Circle: The Challenge of Cosmopolitan Democracy, London, Ithaca, 1996; W. Michael, A Rebel in Defense of Tradition: The Life and Politics of Dwight Macdonald, New York, Basic Books, I994. 
Human Crisis». Alla radice del loro rapporto - forse insieme a quello con Caffi il legame umano e intellettuale più intensamente vissuto da Chiaromonte - vi era una forte identità di vedute sul piano etico, estetico e in parte filosofico. Fu dunque più che naturale per lui assumere le difese di Camus al momento della rottura con Sartre, da cui peraltro lo distanziavano anche gli orientamenti politici, visto il rigoroso anticomunismo che gli derivava da un genuina istanza antitotalitaria ${ }^{15}$. E Sartre, il "comunista impossibile» ritratto nel Tempo della malafede ${ }^{16}$, un pamphlet scritto nel 1953 e dedicato alla fenomenologia della mentalità comunista, divenne, soprattutto negli anni più caldi della Guerra fredda, ai suoi occhi l'esempio più lampante di quella trahison des clercs che vedeva rinnovarsi con l'adesione massiccia o - peggio - il sostegno indiretto degli intellettuali al movimento comunista ${ }^{17}$.

Al secondo lungo soggiorno francese di Chiaromonte - quando di ritorno, nel 1948, in Europa decise di ristabilirsi appunto a Parigi dove rimase fino al 1952 - si deve invece il consolidarsi della sua amicizia con Raymond Aron (la loro conoscenza era infatti più antica e risaliva quanto meno alla fine degli anni Trenta). Di Aron, con cui avrebbe collaborato all'interno delle iniziative promosse dal Congresso per la libertà della cultura e di cui «Tempo presente» avrebbe pubblicato diversi scritti, Chiaromonte ammirava indubbiamente l'ingegno, ma diffidava, pur condividendone spesso le analisi (dai totalitarismi, alla natura della guerra moderna fino al 'maggio parigino' del Sessantotto), dal 'realismo' che ne ispirava le posizioni politiche. E del resto, se guardiamo alla cultura francese, gli autori di Aron - anche se è indubbio riscontrare in Chiaromonte più di un'assonanza con la riflessione dell'Halévy dell'Ère des tyrannies - non sono certo quelli di Chiaromonte. Questi infatti prediligeva Proudhon ${ }^{18}$ (che con Tolstoj e Herzen era per lui uno degli ancestors di un pensiero libertario alternativo a quello egemone nella sinistra europea), Georges Gurvicht (conosciuto attraverso Caffi) e Simone Weil, la cui riflessione sulla storia e sulla forza l'aveva restituito alla vita ${ }^{19}$ dopo i giorni cupissimi della disfatta della Terza Repubblica, segnati anche dalla tragica morte della

15. Chiaromonte analizzerà la rottura fra Camus e Sartre in Paris Letter: Sartre versus Camus, «Partisan Rewiev», vol. XIX, n 6, novembre-dicembre 1952.

I6. N. Chiaromonte, Il tempo della malafede: il comunismo e gli intellettuali, Roma, Associazione italiana per la libertà della cultura, 1953 .

17. Su questi temi mi permetto rimandare a C. Panizza, Nicola Chiaromonte e il tradimento dei chierici, "Annali della Fondazione La Malfa», vol. 26, 2012, pp. 199-216.

18. A questo proposito si veda un suo saggio apparso su "politics», Social Law, after Proudhon, gennaio 1945.

19. Cfr. N. Chiaromonte, L'Iliade di Simone Weil, "Il Mondo», 30 maggio 1953, ora anche in Id., Silenzio e parole. Scritti filosofici e letterari, cit., pp. 213-2I7. 
prima moglie, la pittrice Annie Pohl. Insieme ad Aron, Camus, Malraux - tre figure certo assai rappresentative degli orientamenti prevalenti in seno agli intellettuali francesi appartenenti alla stessa generazione di Chiaromonte (era del 1905) - andrebbero almeno ricordati, fra quelli con cui lui fu più assiduamente in contatto, Alfred Rosmer, Maurice Nadeau, Jean Bloch-Michel, Jean-Pierre Vernant, e — pur se non di nazionalità francese - François Bondy, il direttore di «Preuves», di cui Chiaromonte fu un assiduo collaboratore. E prima ancora andrebbe menzionato il più anziano Paul Desjardins che lo introdusse negli ambienti legati alle Décades de Pontigny ${ }^{20}$, nonché Clara Malraux e Francine Camus, alle quali fu legato da profonda amicizia.

Tutte maglie di una rete di relazioni molto estesa, in parte coincidente, ma non sempre, con quella del Congresso per la libertà della cultura ${ }^{2 r} \mathrm{di}$ cui Chiaromonte era una delle personalità di spicco, e che abbracciava oltre alla Francia e naturalmente all'Italia, il mondo anglosassone, specie gli Stati Uniti, e il variegato universo degli esuli politici dell'est europeo. Fu in particolare il caso dei polacchi gravitanti attorno alla rivista «Kultura» (Gustaw Herling-Grudziński, Czesław Miłosz, Konstanty Jeleński), pubblicata peraltro sempre a Parigi. Risorse di cui naturalmente Chiaromonte si avvalse nella scelta dei collaboratori di «Tempo presente», che, legato al network di riviste del Congresso per la libertà della cultura, ebbe una vocazione spiccatamente internazionale. Per tutti questi motivi - cela va sans dire - la Francia vi ricevette grande attenzione, in primo luogo da

20. Va qui introdotta una precisazione: la decisione assunta da Chiaromonte nell'estate del 1934 di trasferirsi in Francia non fu dettata da immediate motivazioni politiche, come si potrebbe pensare considerata la sua militanza clandestina nella riorganizzazione delle file di "Giustizia e Libertà» a Roma, cui si dedicò al suo rientro in Italia dopo il suo primo soggiorno parigino. A deciderne il ritorno in Francia era stato l'invito ricevuto da Desjardins per partecipare fra gli ascoltatori alle Décades. Solo successivamente, venuto a conoscenza del fatto di essere stato identificato dall'OVRA come collaboratore dei «Quaderni di Giustizia e Libertà», Chiaromonte decise per necessità di stabilirsi permanentemente in Francia. A quell'edizione delle Décades Chiaromonte si recò assieme agli amici Giorgio Diaz de Santillana, che doveva parteciparvi come relatore, Alberto Moravia, Filippo Burzio e Guglielmo Alberti. Cfr. C. Panizza, Nicola Chiaromonte. Una biografia: gli anni della formazione (1905-1940), tesi di dottorato, Università degli studi di Torino, 24 maggio 2007. Sugli appuntamenti di Pontigny cfr. F. Chaubet, Paul Desjardins et les décades de Pontigny, Villeneuve-d'Ascq, Septentrion, I999.

2I. Sul Congresso per la libertà della cultura, P. Coleman, The Liberal Conspiracy. The Congress for Cultural Freedom and the Struggle for the Mind of Postwar Europe, New York, Free Press, 1989; G. Scott-Smith, The Politics of Apolitical Culture. The Congress for Cultural Freedom, the CIA and Post-War American Hegemony, London-New York, Routledge, 2002; P. Grémion, Intelligence de l'anticommunisme. Le Congrès pour la liberté de la culture à Paris, Parigi, Fayard, 1995; F. Stonor Sauders, La guerra fredda culturale. La CIA e il mondo delle lettere e delle arti, Roma, Fazi, 2004 (1999); sui rapporti fra il Congresso e l'Italia, D. Muraca, L'Associazione italiana per la libertà della cultura: il "caso italiano» e il Congress for Cultural Freedom, «Storiografia», vol. II, 2007, pp. I39-I60; P. Carlucci, "Tempo presente» (1956-1968) e il Congress for Cultural Freedom: alcuni appunti per la storia di una rivista, cit.; E. Capozzi, L'opposizione all'antiamericanismo: il Congress for Cultural Freedom e l'Associazione italiana per la libertà della cultura, in P. Craveri e G. Quagliariello, L'Antiamericanismo in Italia e in Europa nel secondo dopoguerra, Soveria Mannelli, Rubettino, 2004, pp. 325-35I. 
parte dello stesso Chiaromonte che puntualmente chiosò lungo gli anni l'evoluzione del panorama politico e culturale francese.

Nei suoi scritti si ritrova anche a distanza di anni una forte empatia per quella che aveva definito ancora nel $196 \mathrm{I}$ "la più amabile delle nazioni»" ${ }^{22}$, da cui ancora ben dopo il 'colpo di stato' del maggio 1958 e in reazione ad esso si attendeva «ven[isse] presto il gesto liberatore che le addita la sua tradizione, e cioè la rinuncia incondizionata a ogni politica di potenza e il rifiuto del principio di autorità in ogni sua forma»" ${ }^{23}$. Ciò avrebbe significato la liquidazione di quel "provincialismo" ammantato di grandeur in cui la società francese era sprofondata, a favore di quella dimensione cosmopolita per Chiaromonte indicatale dalla sua storia migliore. Si sarebbe trattata di una rigenerazione che avrebbe permesso alla Francia di dare un contributo formidabile alla costruzione di uno spazio europeo - culturale e politico - pienamente libero, cui Chiaromonte intensamente aspirava.

Non sarebbe però certo esatto ridurre queste sue reiterate dichiarazioni di solidarietà alla 'Francia civile', a una sorta di idealizzazione, forse un po' romantica, del ruolo che la Francia - e in essa Parigi — aveva svolto e poteva ancora svolgere quale capitale di una ideale repubblica delle lettere europea (o euro-atlantica), nella direzione di un rinnovamento della vita politica, sociale e intellettuale del Vecchio continente. Sarebbe allo stesso modo fuorviante ridurre l'antipatia feroce - peraltro molto diffusa nella stampa e nell'opinione pubblica italiana - che avrebbe riservato al gollismo solo alla frustrazione di qualcuno che vedeva la storia prendere una direzione decisamente contraria a quella auspicata.

In fondo, era innanzitutto una formula polemica questo suo opporre costantemente alla 'Francia eterna' di de Gaulle una ben diversa 'Francia civile', sulla cui vitalità e capacità di aver ragione del torpore che la circondava Chiaromonte si ostinava a confidare, nonostante, come si accennerà, fosse consapevole dei molti elementi che facevano disperare di una rapido superamento della 'crisi francese'.

Era una chiave di lettura apparentemente rigida, dettata innanzitutto dall'urgenza di distanziarsi nettamente dal gollismo, ma che non impedì a Chiaromonte di cogliere, diversamente da molti altri osservatori ita-

22. Commentando, non senza sarcasmo, la riuscita del test nucleare di Reggane, nel deserto algerino, Chiaromonte affermò che i generali, gli unici che potevano rallegrarsi del nuovo status di potenza nucleare acquisito dalla Francia, avevano «il vanto di aver reso odiosa, per la prima volta nella storia, a decine di milioni di uomini, la nazione più amabile della terra» (N. Chiaromonte, Urrà per la Francia, «Tempo Presente», vol. V, $\mathrm{n}^{\mathrm{o}} 2$ 2-3, febbraio-marzo 1960, pp. 186-187).

23. N. Chiaromonte, Il Generale, la Francia e noi, «Tempo presente», vol. V, nº I2, dicembre I960, p. 88I. 
liani, le specificità che non ne facevano una riedizione francese e moderna del fascismo. Negli anni, Chiaromonte si dispose così a registrare, anche grazie a continui, brevi soggiorni parigini, ogni possibile segnale di anticonformismo che potesse agitare quell'inerzia in cui, come abbiamo visto, la Francia - non diversamente dall'Occidente europeo nel suo insieme era a suo dire sprofondata per azione della Guerra fredda. Nel fare ciò egli privilegiò l'evoluzione dei costumi e della mentalità piuttosto che le vicende strettamente politiche. Si trattò di un'analisi condotta sullo sfondo di un'articolata e critica riflessione sulla società di massa e sui cambiamenti che in questo senso la società del benessere veniva dispiegando, a partire dal delinearsi di fenomeni di omologazione culturale storicamente inediti. Avrebbe così registrato per tempo i fermenti giovanili all'origine del Sessantotto ${ }^{24}$.

Va osservato che questa sua convinzione dell'irrilevanza della politique politicienne nel determinare i cambiamenti sostanziali in una società e della necessità di guardare altrove — per comprenderli appieno, non significava necessariamente disinteresse o peggio disprezzo per la politica tout court. Era però fermo nell'idea che l'intellettuale in quanto tale - e non in quanto cittadino - dovesse negarsi a ogni coinvolgimento diretto in essa, al fine di assolvere alla sua funzione di chiarificazione nel campo delle idee. Si trattava di preservare la propria autorevolezza per intervenire nel (e vigilare sul) dibattito pubblico quando la sfera della politica mostrasse di confliggere con quella della libertà della cultura e - in senso lato e più in generale - con il diritto di ciascuno e di tutti all'autonomia della propria coscienza individuale. Giacché in fondo, come aveva più volte ribadito, «il problema vero della politica contemporanea [era] come imporre alla ragione politica le ragioni elementari della morale» ${ }^{25}$.

In questo senso, proprio la vicenda del conflitto algerino e la connessa ascesa al potere di de Gaulle — il 'colpo di stato' del I3 maggio - avevano inevitabilmente suscitato l'interesse appassionato di Chiaromonte. Nel valutare quanto accadeva nel paese nordafricano Chiaromonte aveva assunto la posizione di chi riconosceva come inevitabile l'indipendenza dell'Algeria, ritenendo irrealizzabile qualsiasi altra soluzione, compresa quella federale (distanziandosi così anche dall'amico Camus) ${ }^{26}$. Pur addossando la responsabilità principale del conflitto all'impasse della Quarta

24. Cfr. Id., La rivolta conformista. Scritti sui giovani e il '68, a cura di C. Panizza, Forlì, Una Città-Fondazione Alfred Lewin, Italia, 2009.

25. Id., Importanza del dire di no, "Tempo presente», vol. III, nº II, I958, pp. 893-894.

26. Cfr. Id., Camus e l'Algeria, «Tempo presente», vol. III, nº 7, luglio I958, pp. 587-588. 
Repubblica e all'esercito francese, egli si rifiutava di sposare la causa indipendentista. Sia perché non condivideva l'assolutezza con cui gli indipendentisti algerini — al pari degli oltranzisti francesi — portavano avanti la loro lotta, con l'inevitabile, ma inaccettabile, corollario di fare dell'uso della violenza un obbligo di tipo morale; sia perché un'indipendenza così ottenuta avrebbe condannato l'Algeria a un destino di autarchica indigenza. Come aveva sostenuto Raymond Aron in La tragédie algérienne, entrambe le parti in lotta avevano realisticamente interesse a una soluzione concordata del conflitto, ma ne erano per Chiaromonte impedite dalla logica ideologica che quel conflitto aveva assunto ${ }^{27}$. Nel caso dei generali e della destra più estremista francese essa andava al di là del nazionalismo. Ciò che era in questione in Francia era in fondo il principio secondo il quale uno stato sovrano può e deve usare tutta la forza disponibile per reprimere qualsiasi fermento che ne metta in discussione il potere assoluto in seno alla società. E cioè quel suo preteso «ius imperii, il diritto incondizionato di vita e di morte non solo, ma di disonore, di abiezione e di barbarie che lo Stato moderno non ha ancora cessato di arrogarsi sui cittadini ${ }^{28}$. Era questa la ragione ultima in nome della quale l'esercito francese aveva, secondo Chiaromonte, spianato la strada, non potendo esercitarlo direttamente, al potere quasi monarchico di de Gaulle. Ed essa era anche l'unico nocciolo ideologico della coalizione che sosteneva il Generale, un nocciolo ideologico, peraltro, come proprio il regime nato dal I3 maggio avrebbe dimostrato, indifferente all'ordinamento formale delle istituzioni e sostanzialmente finalizzato, nei fatti, a promuovere l'integrazione dell'esercito nel governo, quale correttivo 'tecnico' al malfunzionamento della democrazia rappresentativa. Per queste ragioni non c'era da attendersi qualcosa di simile a un fascismo francese, nonostante il consenso di cui quella soluzione godeva nell'estrema destra. Ma non vi erano nemmeno motivi per pensare che gli effetti sulla società francese non sarebbero stati comunque dirompenti, giacché quel regime avrebbe comunque aggravato i processi di spoliticizzazione della vita pubblica che già la attraversavano. Anzi, la confusione francese generata dal gollismo e dalla contraddittoria figura di un generale chiamato a mettere al proprio posto l'esercito e i nazionalisti in nome della grandezza nazionale, lasciava intravvedere qualcosa di assai più grave.

Dalla Francia s'è diffuso e continua a diffondersi per l'Europa l'esempio di una violenza razionalmente organizzata e diretta, di un disprezzo metodico dell'uomo e della

27. Id., L'Algeria a lume di ragione, «Tempo presente», vol. III, $\mathrm{n}^{\circ}$ I, gennaio 1958, pp. 73-76.

28. Id., Rifiuto d'obbedienza, «Tempo Presente», vol. V, n 9-10, settembre-ottobre 1960, pp. 715-718. 
convivenza civile, di una volontà di potere senza scrupoli e senza limiti, di un nazionalismo feroce e freddo, più pericoloso assai di quel fascismo contro il quale, in Francia e fuori, si continua bellamente a battagliare ${ }^{29}$.

Giacché non si trattava di riconquistare solo la «legalità» o ristabilire l'«ordine» ma di abbattere dalle «fondamenta la Francia militare e nazionalista» per edificare "una nuova e vera repubblica» ${ }^{\circ}$, Chiaromonte non si faceva in realtà grandi illusioni sulla possibilità di superare rapidamente e positivamente la crisi francese.

Egli ricordava molto bene le amare delusioni del passato, le speranze accese e poi andate deluse sia durante la stagione del Fronte popolare sia durante la Resistenza, quando la mobilitazione sociale in cui si era incarnata la 'Francia civile' era stata a suo parere tradita da quelle forze che ne avrebbero dovuto essere i fedeli interpreti, le organizzazioni partitiche e sindacali. Né egli si aspettava che le cose andassero ora diversamente. Non solo perché lo spettacolo dell'opposizione al gollismo offerto dalla sinistra francese era sconfortante, ma soprattutto per quella sua radicata sfiducia verso ogni forma di partecipazione politica organizzata delle masse.

Ne discendeva la necessità di un lavoro in profondità a sostegno di ogni forma di genuina resistenza al conformismo e all'ambiguità in cui si trovava la vita pubblica in Francia. Si trattava di un compito di chiarificazione cui era chiamato innanzitutto il mondo della cultura e a cui ancora un volta gli intellettuali 'progressisti' — registrava Chiaromonte — si erano perlopiù sottratti. Essi, infatti, in maggioranza avevano operato la scelta di appoggiare la causa del Fln rendendosi così responsabili di rafforzare la logica che condannava il conflitto algerino a non essere risolto, se non attraverso una soluzione di forza. Era ancora una volta una critica alle posizioni di Sartre, accusato peraltro di aver sostituito nel tempo quale soggetto rivoluzionario le classi operaie dei paesi occidentali, ormai non più disponibili a rivoluzioni violente, con le masse diseredate dei paesi in via di decolonizzazione.

Per queste ragioni, Chiaromonte salutò con entusiasmo la Déclaration sur le droit à l'insoumission dans la guerre d'Algerie, il famoso 'manifesto dei I2I' — benché sottoscritto anche da Sartre — a sostegno di coloro che si rifiutavano di prestare servizio militare in Algeria. «Si tratta infatti della prima manifestazione, in un paese dell'Europa non totalitaria, del fermento che ribolle dovunque: segno precursore, forse, di quella ripresa libera della vita politica che la Guerra fredda e i vecchi schemi politici hanno con-

29. Id., La Francia sinistrata, "Tempo Presente», vol. VII, nº 6, giugno I962, pp. 454-455.

3o. Ibid. 
gelato per quindici anni» ${ }^{31}$. Di concerto con Silone, Chiaromonte avviò una nutrita serie d'iniziative di sostegno al Manifesto. Insieme a Alberto Moravia, Guido Piovene, Lionello Venturi e Elio Vittorini indirizzò un telegramma ad André Malraux, all'epoca ministro degli Affari culturali francesi, per protestare contro i procedimenti giudiziari avviati nei confronti dei suoi firmatari. $\mathrm{Su}$ "Tempo Presente» apparve una Dichiarazione di solidarietà ${ }^{22}$, stesa da Chiaromonte e Vittorini, che venne controfirmata da decine di intellettuali, non solo italiani (fra gli altri Dwight Macdonald, Bertrand Russell, Isaiah Berlin e dopo qualche perplessità iniziale la stessa Hannah Arendt) ${ }^{33}$. Con la stessa partecipazione la rivista avrebbe commentato i processi Jeanson, il caso Sagan e prima ancora quello del padre Christian Corre. Le iniziative di Chiaromonte furono peraltro oggetto di critica negli ambienti del Congresso, in particolare da parte del direttore di «Preuves», François Bondy ${ }^{34}$. A dimostrazione dell' assoluto rispetto della libertà intellettuale dei propri collaboratori provato da Silone e Chiaromonte, esse furono criticamente discusse, sulle stesse pagine di «Tempo presente», anche da Bloch-Michel ${ }^{35}$, cui era normalmente affidato il ruolo

3I. Id., Rifiuto d'obbedienza, cit.

32. La Dichiarazione di solidarietà con gli intellettuali francesi apparve con il titolo Diritto alla resistenza, nel numero di novembre 1960 (vol. V, n $^{\circ}$ II, pp. 785-788) di «Tempo presente». I firmatari italiani furono: M. Ageno, F. Albini, E. Amaldi, G. C. Argan, R. Bauer, L. Belgioioso, A. Benedetti, L. Berio, G. Bernardini, P. Bigongiari, R. Bilenchi, C. Bo, L. Borghi, C. Brandi, P. Bucarelli, A. Buzzati-Traverso, I. Calvino, R. Cantoni, A. Capitini, C. Cassola, N. Chiaromonte, F. Compagna, M. Conversi, L. Dallapiccola, E. Emanuelli, E. Enriquez Agnoletti, F. Fellini, G. Ferrata, E. Flaiano, I. Gardella, E. Garin, A. Garofalo, V. Gassmann, P. Grassi, G. Herling, A. Lattuada, G. Levi Della Vita, R. Longhi, M. Luzi, O. Macrì, L. Mazzucchetti, M. Mila, P. Milano, A. Mondadori, G. Montalenti, A. Monteverdi, E. Morante, A. Moravia, E. Morlotti, C. Musatti, C. Nivola, E. Paci, M. Pannunzio, E. Peressutti, G. Piovene, V. Pratolini, S. Quasimodo, C. L. Ragghianti, G. Righini, E. N. Rogers, L. Rognoni, E. Rossi, G. Salvini, G. Santomaso, T. Scialoja, V. Sereni, I. Silone, A. Spinelli, G. Strehler, G. Toraldo di Francia, G. Ungaretti, A. Valli, E. Vedova, L. Venturi, E. Vittorini, B. Zevi; nel Regno Unito: A. J. Ayer, I. Berlin, R. Hoggart, P. Ignotus, D. Jacobsen, B. Russel, J. Russel; negli USA: L. Abel, H. Arendt, E. Bentley, W. Demby, I. Howe, A. Kazin, F. Kline, W. de Kooning, S. Kunitz, M. McCarthy, D. Macdonald, R. Motherwell, E. Nagel, W. Phillips, R. Poggioli, P. Rahv, H. Rosember, M. Rothko, G. de Santillana, M. Schapiro.

33. Chiaromonte e Arendt si conobbero durante la Seconda guerra mondiale a New York, ai tempi della collaborazione di Chiaromonte a "politics» cui Arendt destinò alcuni saggi. Dopo il ritorno in Europa di Chiaromonte, rimasero sempre in contatto, anche grazie alla comune amicizia con la scrittrice Mary McCarthy. In particolare Chiaromonte prese posizione a difesa di Arendt durante la polemica suscitata dai suoi interventi in merito alla vicenda Eichmann. Cfr. l'epistolario fra McCarthy e Arendt, Between Friends: The Correspondence of Hannah Arendt and Mary McCarthy, I949-1975, New York, Harcourt Brace \& Company, 1996. Sul profondo legame filosofico che univa la riflessione di Chiaromonte e Arendt, P. Carlucci, Intellettuali nel Novecento: il confronto di Nicola Chiaromonte con Hannah Arendt, "Ricerche di storia politica", vol. I, 2OII, pp. 3-28. Sulla collaborazione di Arendt a "politics", P. Adamo, "Politics», il radicalismo libertario e Hannah Arendt, in M. Durst e A. Meccariello (a cura di), Hannah Arendt. Percorsi di ricerca tra passato e futuro 19752005, Firenze, Giuntina, 2006, pp. 57-7I.

34. Su questo vedi il cenno che alla vicenda fa P. Carlucci, Intellettuali nel Novecento: il confronto di Nicola Chiaromonte con Hannah Arendt, cit.

35. J. Bloch-Michel, Lettera da Parigi, «Tempo presente», vol. V, nº 9-IO, settembre-ottobre 1960, pp. 703-706. 
di corrispondente da Parigi. Quei malumori dimostravano la posizione peculiare dei due intellettuali italiani in seno al Congresso. Silone e Chiaromonte fin dalla nascita di "Tempo presente» avevano infatti 'contrattato' una maggiore autonomia per la loro rivista dalle linee ufficiali dell'associazione internazionale, al fine di non essere impediti nella loro libertà di muovere critiche alle politiche dei paesi del blocco occidentale. Si trattava di una preoccupazione che non valeva solo per la politica interna italiana, dove non si voleva rinunciare in nome dell'atlantismo a un'irriducibile opposizione ai governi centristi democristiani, ma anche e forse soprattutto per il processo di decolonizzazione.

La fine nel 1962 del conflitto algerino aveva significato solo apparentemente un miglioramento della situazione politica, giacché non ne era affatto derivato quell'indebolimento del potere di de Gaulle che taluni a sinistra si aspettavano. Nonostante le tante critiche che lo investivano e le contraddizioni che lo condannavano, il regime gollista rimaneva inevitabilmente saldo in sella, grazie anche alla debolezza delle opposizioni. La 'Francia civile' - ossia in sostanza quei ceti medi che egli riteneva in realtà favorevoli a una politica democratica —, intimorita dalla possibilità che una caduta del Generale potesse determinare l'avvento al potere dell'estrema destra e condurre direttamente a una dittatura militare e forse alla guerra civile, ed intralciata, a sinistra, da un Partito comunista ridotto a "una vuota impalcatura», aveva continuato a vedere in de Gaulle il «minore dei mali», consentendogli di proseguire in una politica estera che rappresentava sul piano europeo un vulnus gravissimo e per la Francia un'insensatezza. Non per questo Chiaromonte disperava che in Francia «non ci fosse comunque qualcosa d'altro che de Gaulle da una parte e Thorez dall'altra», peraltro a suo dire solo apparentemente contrapposti $\mathrm{i}^{36}$.

Il fatto nuovo, in grado, almeno negli auspici di Chiaromonte, di vincere l'immobilismo della società francese, avrebbe potuto essere il movimento studentesco, del cui fermento - soprattutto negli Stati Uniti, ma anche in Italia e in Francia - aveva colto per tempo, almeno dai primi anni Sessanta, e con favore, le prime avvisaglie. Gli pareva promettente la tendenza dei giovani a mettere in discussione il mondo ereditato dagli adulti, e le culture politiche dominanti, in particolare la comunista e la cattolica (in quest'ultimo caso anche per effetto del processo riformatore apertosi con il Concilio Vaticano II). Era convinto che l'acquisizione di un piena autonomia di giudizio li avrebbe spinti a rifiutare quelle tradizioni 
perché inconciliabili con le loro rivendicazioni di libertà e perché inadeguate alla loro «legittima impazienza a misurarsi con le questioni d'oggi» ${ }^{37}$. Pur con molta cautela, riflettendo soprattutto sul caso italiano e francese, Chiaromonte sperava, almeno al suo esordio, che il movimento studentesco potesse rivelarsi un fattore importante di secolarizzazione della politica oltre che della società. Avrebbe cioè potuto contribuire in prospettiva a liberare, una volta per tutte, la società dalla presa su di essa esercitata dalle grandi narrazioni ideologiche novecentesche. Nel caso francese non era questione solo di comunismo, ma anche di quel residuo di mentalità nazionalista (e statolatrica) diffuso a destra come a sinistra. I fatti del Maggio parigino - pur considerando condivisibili le ragioni della rivolta e l'afflato libertario che la caratterizzava - avrebbero costituito un'ulteriore delusione per Chiaromonte. Al pari, ancora una volta, dell'Aron di La Révolution introuvable, Chiaromonte vi vedeva uno psicodramma collettivo, una ribellione solo apparente che avrebbe definito nei suoi articoli per «La Stampa», cui aveva preso a collaborare proprio nel 1968, dopo la fine dell'esperienza di «Tempo presente», con un ossimoro: «la rivolta conformista ${ }^{38}$. In un duplice senso. Sia perché - ed era stata la chiave del suo successo e della sua estensione globale - quella rivolta, comprensibile come fatto generazionale solo alla luce di quella omologazione culturale contro la quale pure i giovani dichiaravano di volersi ribellare, finiva per produrre un conformismo di tipo nuovo altrettanto oppressivo degli individui rispetto a quello che i giovani intendevano denunziare; sia perché nel loro rifiuto del mondo dei padri i giovani finivano per compiere scelte in continuità con il loro esempio. Sul piano politico essi non facevano infatti nient'altro che radicalizzare e estremizzare le idee ricevute dalle generazioni precedenti, replicando quella assolutizzazione della politica, divenuta nel Novecento un succedaneo della religione, che conduceva ancora una volta alla legittimazione della violenza ${ }^{39}$.

Pur confidando nei cammini individuali che comunque quei giovani prima o poi avrebbero intrapreso e non escludendo - guardando soprattutto al caso americano - che comunque quella ribellione giovanile avrebbe avuto positivi effetti sul lungo periodo, Chiaromonte osservava sul finire

37. Id., Gioventù indocile, "Tempo presente», aprile 1965, pp. 2-5, ora riprodotto anche in Id., La rivolta conformista, cit., pp. 25-33.

38. Id., I giovani e la politica, «Tempo presente», febbraio 1967; ora anche in Id., La rivolta conformista, cit., pp. 50-54.

39. Sulla riflessione di Chiaromonte sul rapporto fra religione e politica e sugli effetti nella società contemporanea dell'obliterarsi della dimensione del 'sacro', cfr. il carteggio con suor Jerome, al secolo Melanie von Nagel, Fra me e te la verità. Lettere a Muskha, a cura di C. Panizza e W. Karpinski, Forlì, Una Città, 2013. 
degli anni Sessanta come per l'ennesima volta - dopo la delusione del Fronte popolare, della guerra civile spagnola e poi della Resistenza - fosse fallito sul nascere (una volta di più anche per responsabilità degli intellettuali) il tentativo di impostare il problema politico nei suoi giusti termini. Per lui infatti il problema essenziale non era il conflitto fra nazioni o fra classi, ma la salvaguardia del pluralismo e della spontaneità della vita associata dal tentativo del potere statale di averne ragione riconducendoli a un qualche astratto principio ordinatore. 